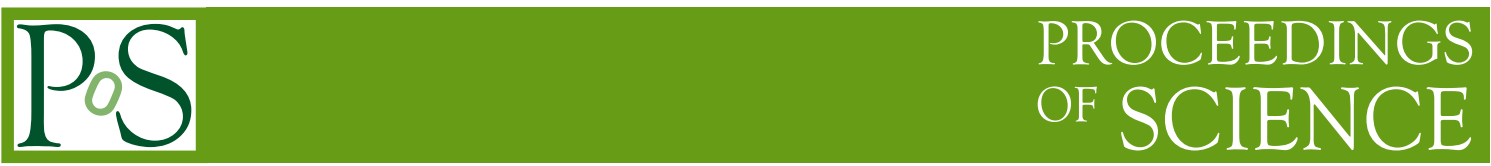

\title{
The X-ray weak state in narrow-line Seyfert 1 galaxies
}

\section{Luigi C. Gallo*}

Department of Astronomy \& Physics, Saint Mary's University, 923 Robie Street, B3H 3C3,

Halifax (Canada)

E-mail: lgallo@ap.smu.ca

\begin{abstract}
Active galaxies in an X-ray weak state, when the direct power law component is significantly diminished, provide the opportunity to study the nature of the other continuum components in unprecedented detail. Narrow-line Seyfert 1 galaxies (NLS1s) are regularly caught in an X-ray weak state and consequently are excellent laboratories to study these underlying spectral components. In this work we explore the general characteristics of X-ray weak AGNs and examine two such objects, PHL 1092 and PG 0844+349, in detail.
\end{abstract}

Narrow-Line Seyfert 1 Galaxies and their place in the Universe - NLS1,

April 04-06, 2011

Milan Italy

\footnotetext{
* Speaker.
} 


\section{High-energy spectral complexity}

With the launch of XMM-Newton came the potential to obtain high signal-to-noise and good resolution spectra of active galaxies in the $0.3-12 \mathrm{keV}$ range. In particular, the early "snap-shot" observations of narrow-line Seyfert 1 galaxies (NLS1s) revealed a plethora of previously unseen characteristics like broad iron emission lines (e.g. [17], [9], [10]), sharp spectral drops at energies above $7 \mathrm{keV}$ (e.g. [1], [2]), and strong spectral curvature above about $2 \mathrm{keV}$ ([20], [11]). The spectral complexity above $2 \mathrm{keV}$ (i.e. the deviation from a canonical power law) could be attributed to either a relativistically blurred emission from the reflector that dominates the spectrum (e.g. [5], [6]) or partial covering of the X-ray source (e.g. [21], [20]).

With repeated observations of the same objects it was realised that the degree of complexity varied from epoch-to-epoch ([12]) and eventually it was recognized that the degree of complexity exhibited by an object was associated with its X-ray flux state. Specifically, when AGN are in an X-ray weak ${ }^{1}$ state they exhibit a higher degree of spectral complexity ([13]). The general idea put forward to describe the X-ray weak state is that we are seeing the AGN when the primary power law emission is significantly diminished. Observing AGN in this state provides the opportunity to study the underlying spectral components (e.g. the reflected emission and ionised emitter) in unprecedented detail (e.g. [15], [7], [8], [24]).

\section{Characteristics of NLS1 in an X-ray weak state}

The original work demonstrating spectral complexity in the X-ray weak state was based on a small sample of NLS1s ([13]). That work is currently being expanded to include a much larger sample of both NLS1s and broad-line Seyfert 1 galaxies (BLS1s). Preliminary results are discussed here and general characteristics of the sample are also discussed by Vasudevan ([23]). To summarize, $\mathrm{X}$-ray weakness is determined from comparing $\alpha_{o x}$, which is the power law connecting the $2500 \AA$ and $2 \mathrm{keV}$ flux. The UV and X-ray data are observed simultaneously using the Optical Monitor and the EPIC-pn, respectively, on XMM-Newton.

In Figure $1, \Delta \alpha_{o x}$, which is the difference between the measured $\alpha_{o x}$ and that expected from the $L_{U V^{-}} \alpha_{o x}$ relation specific to our sample, is plotted as a function of $\mathrm{H} \beta$ line width $(F W H M)$. The sample shows significant scatter, but demonstrates that AGNs with $F W H M<2000 \mathrm{~km} \mathrm{~s}^{-1}$ (i.e. NLS1s) exhibit X-ray weak states often. The reason is perhaps linked to the fact that NLS1s appear to exhibit larger amplitude X-ray variations than BLS1s, thus more capable of plunging into a low state. However, exactly why NLS1s are more extremely variable is still an open question. Nonetheless, this demonstrates that NLS1s are excellent laboratories to study X-ray weak states in AGNs.

Using the simultaneous X-ray and UV data, each observation in the sample was fitted with a multicolour disc blackbody and power law to identify the variable component leading to the weak state. Objects for which multi-epoch data existed showed very little difference in the blackbody disc temperature and flux from epoch-to-epoch (Figure 2). This was the case for both NLS1s and BLS1s suggesting that X-ray weakness is not normally driven by changes in the blackbody component.

\footnotetext{
${ }^{1} \mathrm{X}$-ray weakness is in reference to the expected X-ray flux of an object based on its UV luminosity, and it is quantifi ed by the term $\alpha_{b x}$.
} 


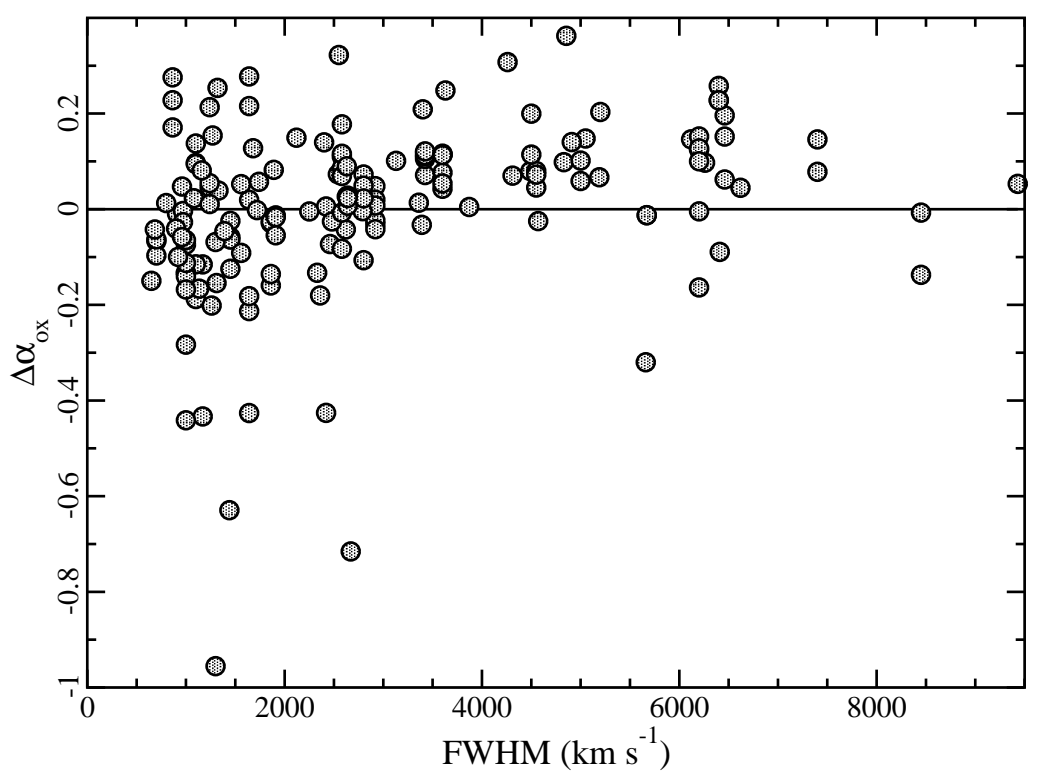

Figure 1: $\Delta \alpha_{o x}$, which is the difference between the measured $\alpha_{o x}$ and that expected from the $L_{U V}-\alpha_{o x}$ relation specific to our sample is plotted as a function of $\mathrm{H} \beta$ line width $(F W H M)$. The more negative the value is the more X-ray weak an object is. NLS1s exhibit X-ray weakness more often than BLS1s. Note that many of the points correspond to the same objects observed at different epochs (not illustrated in this particular figure). The $\alpha_{o x}$ variability in a given object can be examined and upon doing so it does appear the amplitude of the variations is larger in NLS1s.

Figure 2 also demonstrates how NLS1s typically are associated with higher disc temperatures. This could be associated with lower black hole masses in NLS1s, but could also be reconciled if the disc inner radius were smaller in NLS1s. A smaller disc radius would also explain the need for the extreme relativistic effects often seen in NLS1s (e.g. [7], [8]).

The power law component exhibits more obvious changes for objects in a low X-ray state (Figure 3). On average, NLS1s do appear to possess steeper photon indices, but both BLS1s and NLS1s show flatter spectra in the low state. This is also accompanied by significant changes in the brightness of the power law component. For both NLS1s and BLS1s the origin of the X-ray weakness appears to be tied to changes in the power law component and not the accretion disc.

\section{Observations of NLS1 in an X-ray weak state}

In Section 2 we described preliminary work from an analysis of the X-ray weak state in a sample of AGNs. In this section we describe detailed observations of a few individual objects that have been examined and chase after the cause of the changes in the power law component. The power law originating from the corona could be changing in intensity or shape for a number of reasons. For example, a cooling of the corona, or changes in the magnetic field, or ejection of the corona from the system. These would constitute as intrinsic changes in the luminosity or shape of the power law due to physical changes in the corona. Other effects could be geometrical, for 


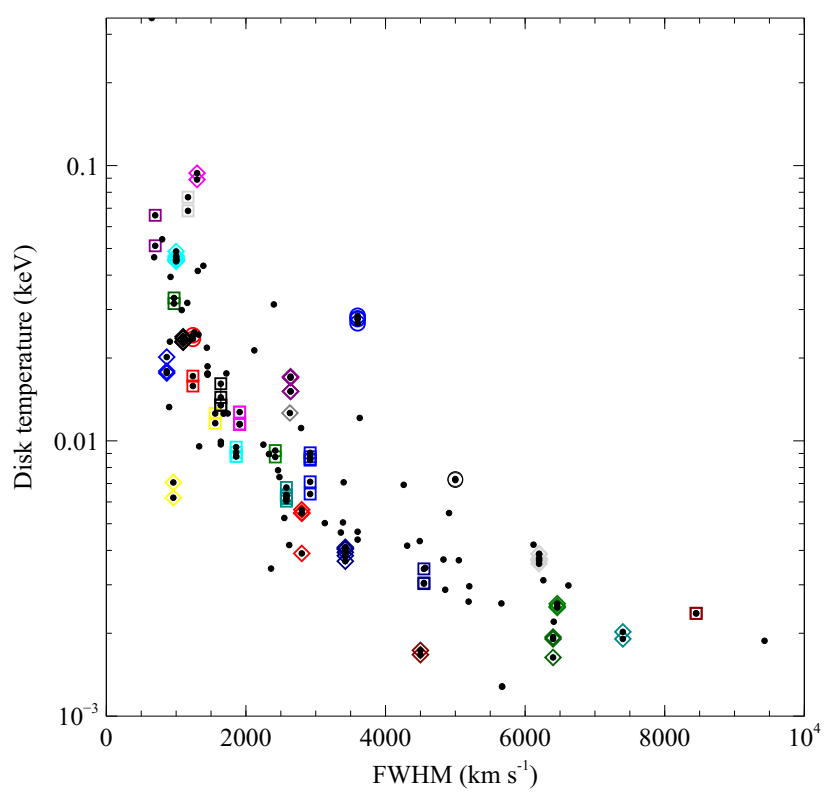

Figure 2: The disc temperature plotted against $\mathrm{H} \beta$ line width. On average, NLS1s $(F W H M<2000 \mathrm{~km}$ $\mathrm{s}^{-1}$ ) exhibit higher disc temperatures than BLS1s. Multi-epoch temperatures obtained for the same AGN are shown in colour. These show very little change in temperature from one epoch to another.

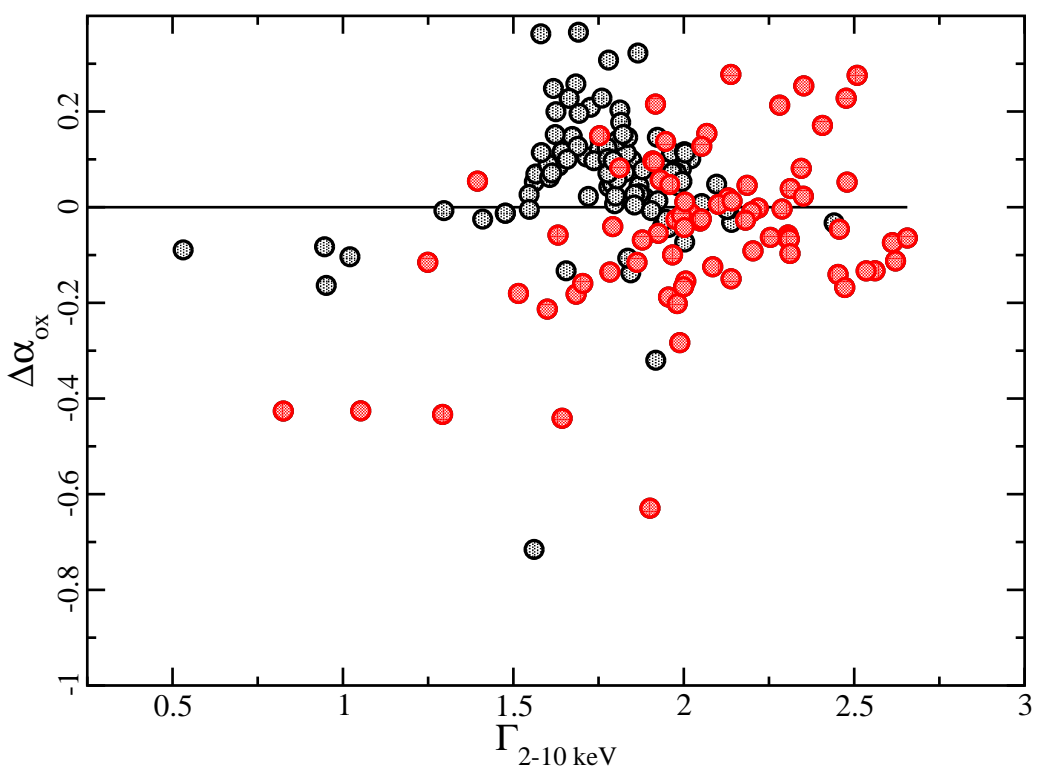

Figure 3: $\Delta \alpha_{o x}$ (same as in Figure 1) is plotted against the photon index of the $2-10 \mathrm{keV}$ power law. On average, NLS1s (red) have steeper spectra than BLS1s (black), but in X-ray weak states both types of Seyferts exhibit fht spectra. 


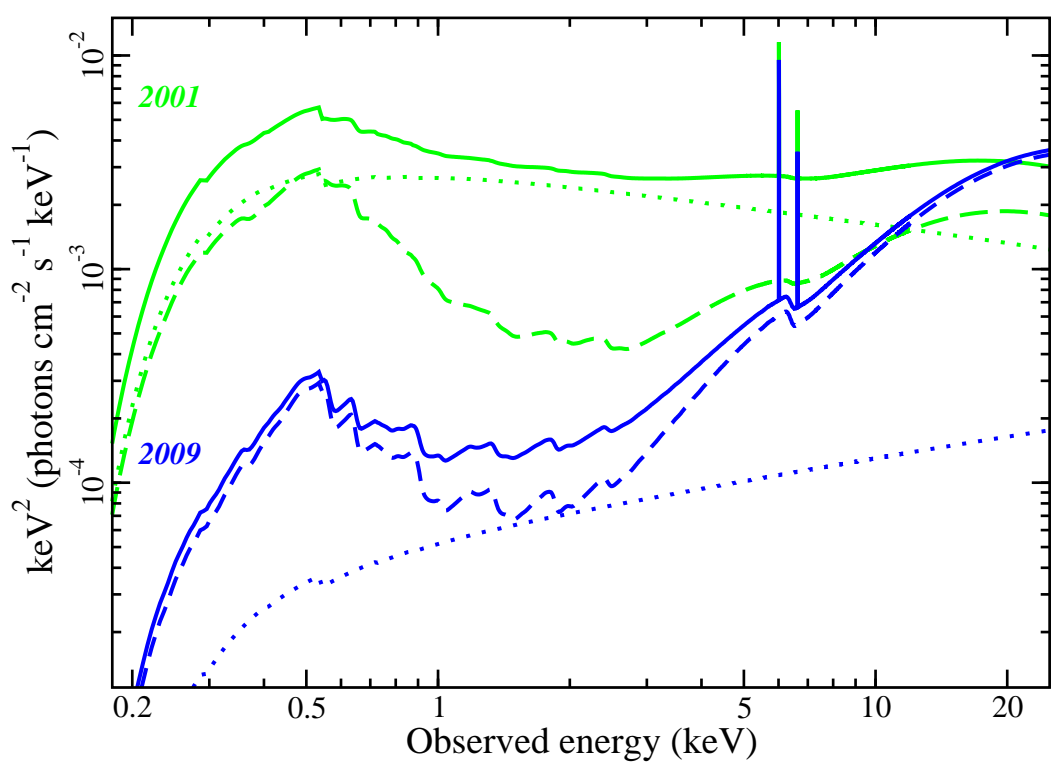

Figure 4: Decomposition of the PG 0844+349 spectral model in the 2001 high-flux state (green) and the 2009 low-flux state (blue). The most obvious change between the two spectra is the diminishing of the power law component (dotted curve) relative to the reftection component (dashed curve) during the low-flux state. In 2009 (solid blue curve) the spectrum was dominated by the reftection component whereas in 2001 (green solid curve) the spectrum was power law dominated. Adapted from [14].

example due to light-bending close to the black hole (e.g. [18]). In this case, the observer would see differences in the brightness or hardness of the power form without there being actual intrinsic changes in the coronal emission. In this section we discuss how both scenarios are viable in NLS1s.

\subsection{PG 0844+349}

PG $0844+349$ is a well-studied AGN that has been observed with XMM-Newton at three separate epochs in 2000, 2001, and 2009. In the earlier observations the source was in a relatively bright state and the X-ray spectrum was dominated by the power law component. However, in 2009 the object was caught in low-flux state and the X-ray spectrum took on a much more complicated appearance that was consistent with being reflection dominated ([8]). Moreover, it is noteworthy that the UV emission was comparable in both the high- and low-flux states. PG $0844+349$ was in an X-ray weak state in 2009.

The three observations could be fitted in a self-consistent manner if the significant X-ray variability were attributed mostly to the diminishing of the power law component during the low-flux state (Figure 4) ([14]). In this interpretation the power law component diminished in intensity due to light bending effects that do not require significant physical changes in the structure of the corona.

The light-bending scenario is a rather common interpretation for the behaviour of NLS1s (e.g. [凹). It adequately describes the rapid, large amplitude variations in NLS1s and the spectral be- 

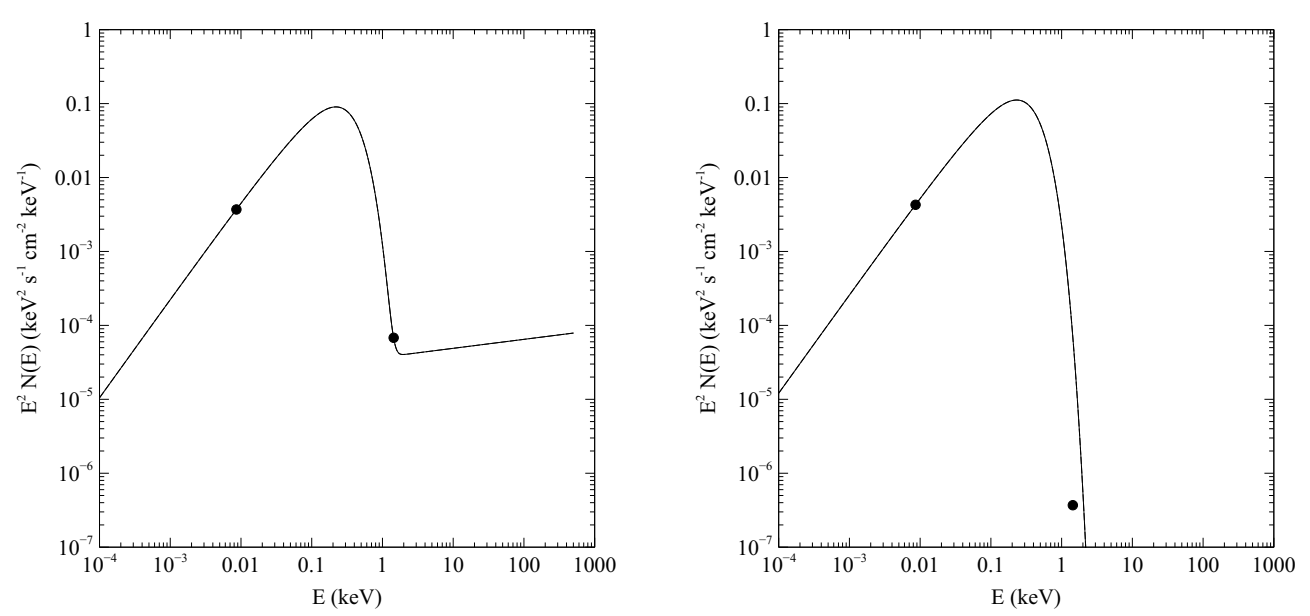

Figure 5: The UV-to-X-ray spectral energy distribution (SED) for PHL 1092 in 2003 (left) and 2008 (right). All the X-ray data are used but only the $2 \mathrm{keV}$ data point is shown in the figure. The model is composed of a disc blackbody and power law. In 2008, the power law component is negligible, but the disc component is sufficient to describe the UV and X-ray data.

haviour in a self-consistent manner. However, it does not describe the low state of all objects.

\subsection{PHL 1092}

PHL 1092 is a radio-quiet quasar exhibiting extreme NLS1 behaviour. It was recognised to display extreme variations in the X-rays during a 20-day monitoring campaign with ROSAT ([3]). An XMM-Newton snap-shot observation in 2003 revealed a complex spectrum showing significant curvature and a strong soft-excess ([11]). The extreme behaviour could be understood in terms of light-bending effects (e.g. [18]), but detailed modelling was not possible with the short observation. A deep pointed observation five years later in 2008 intended to study the light-bending scenario in detail revealed a surprise when the X-ray flux had dropped by more than two orders of magnitude ([19]). UV data from the Optical Monitor and a contemporaneous optical spectrum showed that the optical/UV continuum and emissions lines were comparable to previous observations.

PHL 1092 is one of the objects in the sample described in Section 2. On fitting the UV and X-ray data from both epochs with a disk blackbody and power law we find that the blackbody component is constant at both epochs, but the power law has effectively disappeared in the 2008 low state (Figure 5). Such extreme changes could not be attributed to light-bending effects, but must be associated with intrinsic changes in the corona.

\section{Conclusions}

The X-ray weak state in AGN produces a unique opportunity to study the underlying physical components in the AGN that are normally overwhelmed by the primary power law emission. It also provides an opportunity to examine the origin of the coronal variability. X-ray weakness is rather common among NLS1s making them ideal systems to study the low-flux state. Why this is the case 
for NLS1s more so than for BLS1s is not certain, but could simply be related to the typically larger amplitude X-ray variability exhibited by NLS1s.

The dominant mechanism for power law weakness in NLS1s appears to be a result of light bending close to the black hole. While the direct power law emission is isotropic and relatively constant in the source frame, at times the observer may see less of this direct emission as it is bent back toward the gravitation potential of the black hole system. Other models, such as partial covering, have not been ruled out in all cases (e.g. [16], [22]), but the very rapid variability is always a challenge to understand in those models ([8]). While light bending is a favourable explanation for X-ray weakness in NLS1s it is certainly not always applicable (e.g. [19], [16]). There are examples where the power law continuum source has undergone some physical change.

\section{Acknowledgments}

Many thanks to my collaborators over the years, most recently A. Fabian, D. Grupe, S. Komossa, G. Miniutti, D. Robertson, N. Schartel, and R. Vasudevan.

\section{References}

[1] T. Boller et al. XMM-Newton discovery of a sharp spectral feature at $7 \mathrm{keV}$ in the narrow-line Seyfert 1 galaxy 1 H 0707 - 49, 2002, MNRAS 343, 89.

[2] T. Boller et al. XMM-Newton spectral properties of the narrow-line Seyfert 1 galaxy IRAS 13224 3809, 2003, MNRAS 343, 89.

[3] W. N. Brandt et al. ROSAT High-Resolution Imager monitoring of extreme X-ray variability in the narrow-line quasar PHL 1092, 1999, MNRAS 303, 53.

[4] J. Crummy J. et al. An explanation for the soft X-ray excess in active galactic nuclei, 2006, MNRAS $365,1067$.

[5] A. C. Fabian et al., How the X-ray spectrum of a narrow-line Seyfert 1 galaxy may be reflection-dominated, 2002, MNRAS 331, 35.

[6] A. C. Fabian et al., X-ray reflection in the narrow-line Seyfert 1 galaxy 1 H 0707-495, 2004, MNRAS $353,1071$.

[7] A. C. Fabian et al. Broad line emission from iron $K$ - and L-shell transitions in the active galaxy 1H0707-495, 2009, Nature 459, 540.

[8] A. C. Fabian: An X-ray view of narrow-line Seyfert 1 galaxies. In: Proceedings of the Workshop Narrow-Line Seyfert 1 Galaxies and Their Place in the Universe, POS (NLS1) 016(2011).

[9] L. C. Gallo et al. I Zw 1 observed with XMM-Newton . Low-energy spectral complexity, iron lines, and hard X-ray flares, 2004, A\&A 417, 29.

[10] L. C. Gallo et al. The narrow-line quasar NAB 0205+024 observed with XMM-Newton, 2004, MNRAS 355,330 .

[11] L. C. Gallo et al. An intense soft excess and evidence for light bending in the luminous narrow-line quasar PHL 1092, 2004, MNRAS 352, 744.

[12] L. C. Gallo et al. Long-term spectral changes in the partial-covering candidate narrow-line Seyfert 1 galaxy 1H 0707-495, 2004, MNRAS 353, 1064. 
[13] Gallo, L. C. Investigating the nature of narrow-line Seyfert 1 galaxies with high-energy spectral complexity, 2006, MNRAS 368, 479.

[14] L. C. Gallo et al. The quasar PG 0844+349 in an X-ray weak state, 2011, MNRAS 412, 161.

[15] D. Grupe et al., XMM-Newton Observations of the Narrow-Line Seyfert 1 Galaxy Mrk 335 in a Historical Low X-Ray Flux State, 2008, ApJ 681, 982

[16] D. Grupe et al., First Detection of Hard X-Ray Photons in the Soft X-Ray Transient Narrow-Line Seyfert 1 Galaxy WPVS 007: The X-Ray Photon Distribution Observed by Swift, 2008, ApJ 136, 2343.

[17] A. L. Longinotti et al. The complex FeK line of the Narrow-Line Seyfert 1 galaxy IRAS 13349+2438, 2003, A\&A 410, 471.

[18] G. Miniutti \& A. C. Fabian, A light bending model for the X-ray temporal and spectral properties of accreting black holes, 2004, MNRAS 349, 1435.

[19] G. Miniutti .et al., PHL 1092 as a transient extreme X-ray weak quasar, 2009, MNRAS 396, 85.

[20] K. Pounds .et al., Exploring the complex X-ray spectrum of NGC 4051, 2004, MNRAS 350, 10.

[21] Y. Tanaka .et al., Partial Covering Interpretation of the X-Ray Spectrum of the NLS1 1H 0707-495, 2004, PASJ 56, 9.

[22] T. J. Turner: X-ray signatures of circumnuclear gas in AGN. In: Proceedings of the Workshop Narrow-Line Seyfert 1 Galaxies and Their Place in the Universe, POS (NLS1) 018 (2011).

[23] R. Vasudevan: Variability, optical-to-X-ray slope and accretion disc properties of a sample of Seyfert 1s. In: Proceedings of the Workshop Narrow-Line Seyfert 1 Galaxies and Their Place in the Universe, POS (NLS1) 007 (2011).

[24] A. Zoghbi: X-ray reverberation delays in NLS1s. In: Proceedings of the Workshop Narrow-Line Seyfert 1 Galaxies and Their Place in the Universe, POS (NLS1) 020(2011). 